\title{
Metabolism and Transport of Maternal Serine by the Ovine Placenta: Glycine Production and Absence of Serine Transport into the Fetus
}

\author{
RUSSELL R. MOORES, JR., CHRISTINE C. TH. RIETBERG, FREDERICK C. BATTAGLIA, \\ PAUL V. FENNESSEY, AND GIACOMO MESCHIA \\ Division of Perinatal Medicine, Departments of Pharmacology, Physiology, and Pediatrics, University of \\ Colorado School of Medicine, Denver, Colorado 80262
}

\begin{abstract}
The present study compares the transplacental transport of $\mathrm{L}-\left[\mathrm{l}-{ }^{13} \mathrm{C}\right]$ serine and $\mathrm{L}-\left[\mathrm{l}-{ }^{13} \mathrm{C}\right]$ leucine in sheep. An in vivo preparation using twin gestations was set up such that the arterial circulation to one uterine horn, including its placenta and fetus, was infused with tracer serine and leucine while the umbilical circulations of both fetuses were sampled. Uterine and umbilical blood flows were measured in each horn. Plasma serine enrichments were $14.6 \pm 2.7 \%$ and $4.3 \pm 1.6 \%$ in the uterine veins draining the experimental and control horns, respectively. Fetal plasma leucine enrichments in the umbilical veins were 50 and $55 \%$ of the uterine venous enrichments in the control and experimental fetuses, respectively. By contrast, during $\mathbf{2 8 0} \mathrm{min}$ of infusion, there was no detectable serine enrichment in either fetal circulation. However, significant plasma glycine enrichment was present in the fetal circulation of the experimental horn and venous glycine enrichments in the experimental horn were significantly greater than arterial glycine enrichments for both the umbilical ( $p$ $<0.02)$ and uterine $(p<0.001)$ circulations. We conclude that under conditions in which leucine transport is easily demonstrable there is no significant transplacental transport of maternal serine and that maternal plasma serine is used within the uteroplacental tissues for producing glycine, some of which is delivered into the fetal circulation. (Pediatr Res 33: 590-594, 1993)
\end{abstract}

\section{Abbreviations}

SHMT, serine hydroxymethyltransferase

The fetal protein accretion associated with fetal growth requires that at least the essential amino acids be transported across the placenta into the umbilical circulation. In contrast, the fetal demand for the nonessential amino acids may be met by any combination of placental transport and/or synthesis within the fetus and placenta. In adult metabolism, it has been clearly demonstrated that there is organ-specific production and utilization of serine and glycine (1). Studies of the net uterine and fetal uptakes of glycine and serine in ovine pregnancy have yielded somewhat confusing results. On the one hand, a net uterine uptake of serine from the maternal circulation has been found (2) without any net flux of serine into the fetal circulation

Received November 23, 1992; accepted February 4, 1993.

Correspondence and reprint requests: Frederick C. Battaglia, M.D., University of Colorado School of Medicine, Department of Pediatrics, Division of Perinata Research, 4200 East Ninth Ave., Box B-199, Denver, CO 80262.

Supported by NIH Program Project Grant HD-00781 and NIH Grant HD01866 .
(3). On the other hand, there is a large umbilical uptake of glycine (4) without measurable uterine uptake of glycine (2, 5, 6). However, the absence of a measurable net uterine uptake of glycine is not convincing due to the high uterine blood flow of pregnancy and maternal plasma glycine concentration. Even a $2 \%$ coefficient of extraction across the uterine circulation, which would not be easy to detect, would represent an appreciable uterine uptake of glycine. These data suggest the possibility that serine derived from the maternal plasma pool may be used within the placenta for glycine production and that this may contribute substantially to the glycine supply to the fetus from the placenta. Recent studies in our laboratory using tracer serine infusion into the fetal circulation have shown that in both late gestation (7) and midgestation (8), fetal plasma serine is utilized within the placenta for glycine production.

The present study was designed to test the hypothesis that maternal serine contributes to the fetal amino acid pool primarily through serine to glycine conversion in the uteroplacental tissues. This study uses a new stable isotope infusion technique in twin pregnancies such that the uteroplacenta of one twin is selectively exposed to high $\left[1-{ }^{13} \mathrm{C}\right]$ serine and $\left[1-{ }^{13} \mathrm{C}\right]$ leucine concentrations via the maternal circulation while the appearance of leucine, serine, and glycine in the fetal circulation of both twins is monitored. Leucine transport and metabolism have been well described in late gestation ovine pregnancy $(9-11)$ and thus can serve as a useful reference point in interpreting the transfer of other amino acids. The animal model used in these studies uses one uterine horn and its fetus as a control in that glycine production from serine in maternal tissues such as the maternal liver will be presented with equal enrichment to both uterine horns, whereas serine enrichment will be higher in the arterial blood perfusing the infused, experimental horn. By this approach, uteroplacental glycine production from serine can be distinguished from glycine production elsewhere in the maternal organism.

\section{MATERIALS AND METHODS}

Biologic preparation. Three pregnant twin gestation ColumbiaRambouillet ewes were studied at approximately $126 \mathrm{~d}$ gestation $($ term $=147 \mathrm{~d})$. After an overnight fast, surgery was performed under pentobarbital sedation and spinal anesthesia with tetracaine. The preparation developed was such that the placenta of one twin (experimental fetus) could be exposed selectively to a high concentration of tracer through local uterine artery infusion (Fig. 1). Polyvinyl catheters used for sampling the fetal circulation were placed into the common umbilical vein and an external iliac artery of each fetus. Catheters were also placed into a fetal brachial vein for infusion of blood flow indicators into each fetus $\left({ }^{3} \mathrm{H}_{2} \mathrm{O}\right.$, ethanol and/or antipyrine). Previous studies have shown no significant differences among these indicators for measure- 
MATERNAL SYSTEMIC CIRCULATION

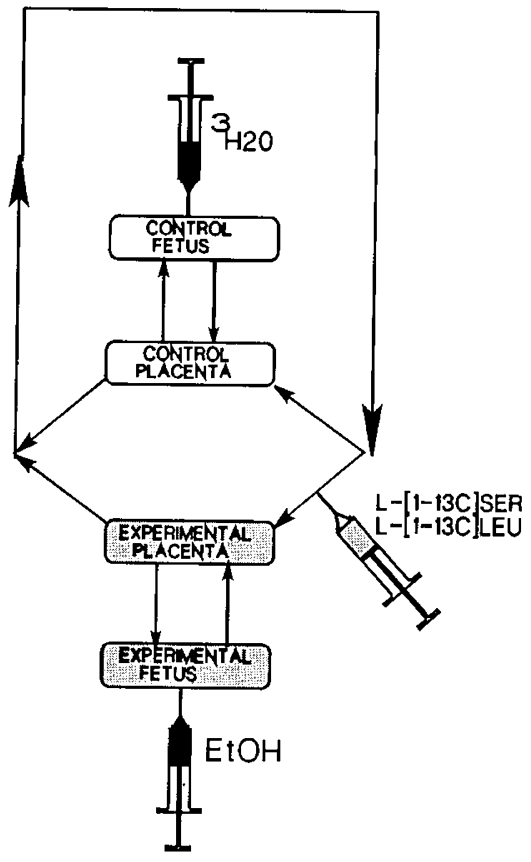

Fig. 1. Scheme of the experimental preparation that selectively exposes the placenta of one twin fetus (experimental placenta) to a high concentration of tracer serine and leucine.

Table 1. Fetal age, fetal weight, umbilical blood flow, uterine blood flow, and fetal oxygen consumption*

\begin{tabular}{lcc}
\hline & Experimental & Control \\
\hline Fetal age $(\mathrm{d})$ & $126 \pm 3$ & $126 \pm 3$ \\
Fetal wt $(\mathrm{kg})$ & $2.31 \pm 0.1$ & $2.35 \pm 0.1$ \\
Umbilical blood flow $(\mathrm{mL}$. & $244 \pm 14$ & $219 \pm 24$ \\
$\quad \mathrm{~min}^{-1} \cdot \mathrm{kg}$ fetus & \\
Uterine blood flow $\left(\mathrm{mL} \cdot \mathrm{min}^{-1}\right)$ & $701 \pm 142$ & $700 \pm 130$ \\
Fetal oxygen consumption & $344 \pm 26$ & $408 \pm 46$ \\
$\quad\left(\mu \mathrm{mol}^{-1} \mathrm{~min}^{-1} \cdot \mathrm{kg} \mathrm{fetus}^{-1}\right)$ & & \\
\hline
\end{tabular}

* Values are means \pm SEM. In each case, $n=3$.

ments of umbilical blood flow (12). Catheters for sampling the maternal circulation were inserted into both uterine veins (experimental and control horns) and one maternal femoral artery. A catheter for infusion of the ${ }^{13} \mathrm{C}$-labeled amino acids was inserted and advanced $1 \mathrm{~cm}$ downstream into a branch of the uterine artery supplying blood to the placenta of only one fetus. Amniotic catheters were inserted for antibiotic administration. The catheters were tunneled s.c. to a pouch on the ewe's flank. After surgery, the ewes were maintained in individual crates and allowed an ad libitum diet of alfalfa pellets, water, and mineral supplements.
Each catheter was irrigated daily with heparinized saline. An intraamniotic injection of $500 \mathrm{mg}$ of ampicillin was administered daily for $3 \mathrm{~d}$ after surgery.

Experimental protocol. The studies were performed 3 to $5 \mathrm{~d}$ after surgery when the animals were fully recovered and eating normally. Studies were performed with the animals conscious and allowed full access to food and water. An infusate consisting of $750 \mathrm{mg}$ of $\mathrm{L}-\left[1-{ }^{13} \mathrm{C}\right]$ serine and $500 \mathrm{mg}$ of $\mathrm{L}-\left[1-{ }^{13} \mathrm{C}\right]$ leucine was prepared in $100 \mathrm{~mL}$ of sterile saline solution. This solution was infused into the uterine artery catheter for approximately $5 \mathrm{~h}$ resulting in infusion rates of 5.2 and $3.3 \mu \mathrm{mol} / \mathrm{min}$ for labeled serine and leucine, respectively. Two solutions, each containing one of the flow indicators, were prepared in sterile saline for determination of uterine and umbilical blood flows in each uterine horn and its fetus. These solutions were infused simultaneously into the experimental and control fetuses at a constant rate for the duration of the study. With this model, the concentrations of $\left[1-{ }^{13} \mathrm{C}\right]$ serine and $\left[1{ }^{13} \mathrm{C}\right]$ leucine reaching the placenta of the experimental twin through the local uterine artery infusion would be significantly greater than that reaching the placenta of the control twin, because in the latter case it is diluted by the maternal amino acid pool. At the same time, uterine and umbilical blood flows could be separately determined in each uterine horn.

Before the start of the infusion, time zero samples were obtained from each catheter. After allowing 170 to $180 \mathrm{~min}$ for the attainment of a steady state, five sets of blood samples were drawn simultaneously from each catheter at approximately 25 min intervals.

After completion of the experiment, each ewe was given a rapid i.v. infusion of euthanasia solution. At autopsy, the weights of each fetus and placenta were obtained, and the positions of the catheters were confirmed.

Chemical analysis. Blood samples for blood gas analysis were collected in glass capillary tubes lined with dried heparin. $\mathrm{Hb}$ concentration expressed as oxygen capacity and oxygen saturation was measured with a Radiometer (Copenhagen, Denmark) hemoximeter. Oxygen content was calculated as the product of the $\mathrm{Hb}$ and the oxygen saturation.

Blood for mass spectral isotope ratio analysis, amino acid analysis, ethanol, ${ }^{3} \mathrm{H}_{2} \mathrm{O}$, and antipyrine concentrations were collected in plastic syringes lined with dried EDTA. Ethanol (13), ${ }^{3} \mathrm{H}_{2} \mathrm{O}$ (14), and antipyrine (15) concentrations were determined as previously described. Plasma for amino acid and mass spectral analysis was immediately stored at $-70^{\circ} \mathrm{C}$.

Amino acid concentrations were determined as previously reported using a JEOL-200A amino acid analyzer (JEOL USA, Cranford, NJ) with norleucine as the internal standard (16). The mass spectral isotope ratio analysis of serine, glycine, and leucine was performed as described previously (4).

Calculations. Umbilical and uterine blood flows were calculated using the steady state diffusion method with ethanol, antipvrrine, and ${ }^{3} \mathrm{H}_{2} \mathrm{O}$ as the blood flow indicators (17). For umbilical blood flow to each placenta:

Table 2. Serine, glycine, and leucine plasma concentration and MPE*

\begin{tabular}{lccccccc}
\hline & $\mathrm{A}$ & $\mathrm{V}_{\mathrm{c}}$ & $\mathrm{V}_{\mathrm{e}}$ & $\mathrm{a}_{\mathrm{c}}$ & $\mathrm{v}_{\mathrm{c}}$ & $\mathrm{a}_{\mathrm{e}}$ & $\mathrm{v}_{\mathrm{e}}$ \\
\hline Leucine & & & & & & & \\
Concentration $(\mu \mathrm{M})$ & $205.8 \pm 37.6$ & $178.6 \pm 36.4$ & $181.4 \pm 39.8$ & $216.3 \pm 50.3$ & $252.1 \pm 60.9$ & $211.1 \pm 40.4$ & $233.9 \pm 41.8$ \\
MPE & $3.3 \pm 1.1$ & $3.4 \pm 1.2$ & $5.8 \pm 0.5$ & $1.5 \pm 0.5$ & $1.7 \pm 0.4$ & $2.6 \pm 0.2$ & $3.2 \pm 0.3$ \\
Serine & & & & & & \\
Concentration $(\mu \mathrm{M})$ & $50.5 \pm 4.5$ & $46.8 \pm 3.8$ & $54.3 \pm 6.3$ & $817.4 \pm 77.8$ & $789.9 \pm 60.4$ & $704.8 \pm 99.3$ & $699.0 \pm 109.7$ \\
MPE & $4.2 \pm 1.4$ & $4.3 \pm 1.6$ & $14.6 \pm 2.7$ & $0.0 \pm 0.0$ & $0.0 \pm 0.0$ & $0.0 \pm 0.0$ & $0.1 \pm 0.1$ \\
Glycine & & & & & & & \\
Concentration $(\mu \mathrm{M})$ & $420.9 \pm 27.9$ & $416.6 \pm 40.8$ & $412.8 \pm 38.0$ & $669.7 \pm 69.3$ & $693.4 \pm 86.7$ & $507.0 \pm 35.1$ & $517.0 \pm 38.4$ \\
MPE & $0.4 \pm 0.04$ & $0.4 \pm 0.04$ & $0.8 \pm 0.1$ & $0.01 \pm 0.02$ & $0.05 \pm 0.02$ & $0.2 \pm 0.05$ & $0.5 \pm 0.05$ \\
\hline
\end{tabular}

*Values are means \pm SEM. A, maternal femoral artery; $V_{c}$, uterine vein of control horn; $V_{e}$, uterine vein of experimental horn; $a_{c}$ and $v_{c}$, umbilical artery and umbilical vein, respectively, of control fetus; $a_{e}$ and $v_{e}$, umbilical artery and umbilical vein, respectively, of experimental fetus; MPE, molar percent enrichment. 
PLASMA SERINE ENRICHMENTS IN BOTH UTERINE HORNS
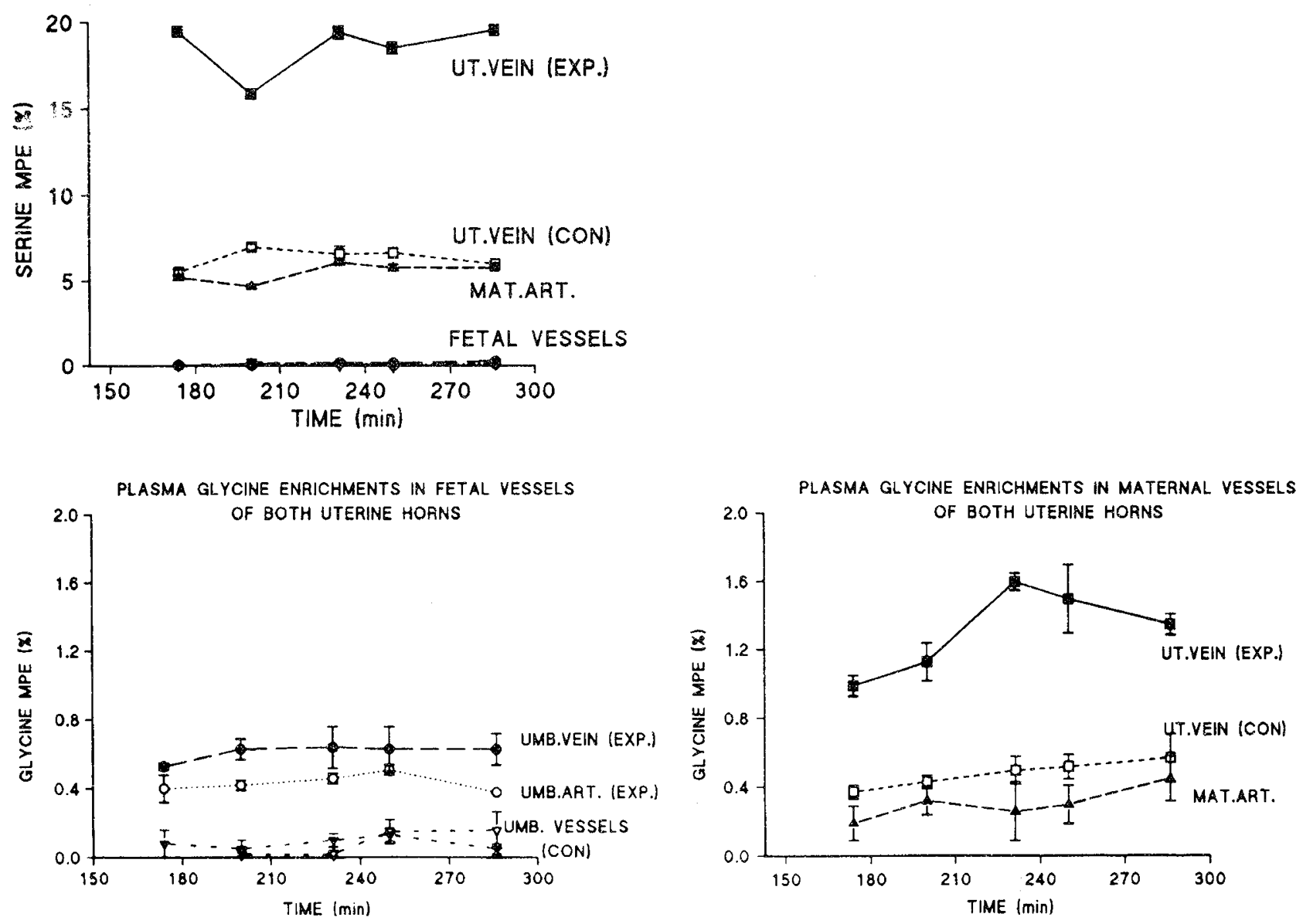

Fig. 2. Serine and glycine enrichments in maternal and fetal plasma during continuous infusion of tracer serine in a uterine artery supplying blood to the experimental placenta. The points labeled MAT.ART represent samples drawn from the maternal femoral artery, i.e. systemic arterial blood upstream from the point of infusion. MPE, molar percent enrichment; $U T$, uterine; EXP, experimental; CON, control; UMB, umbilical.

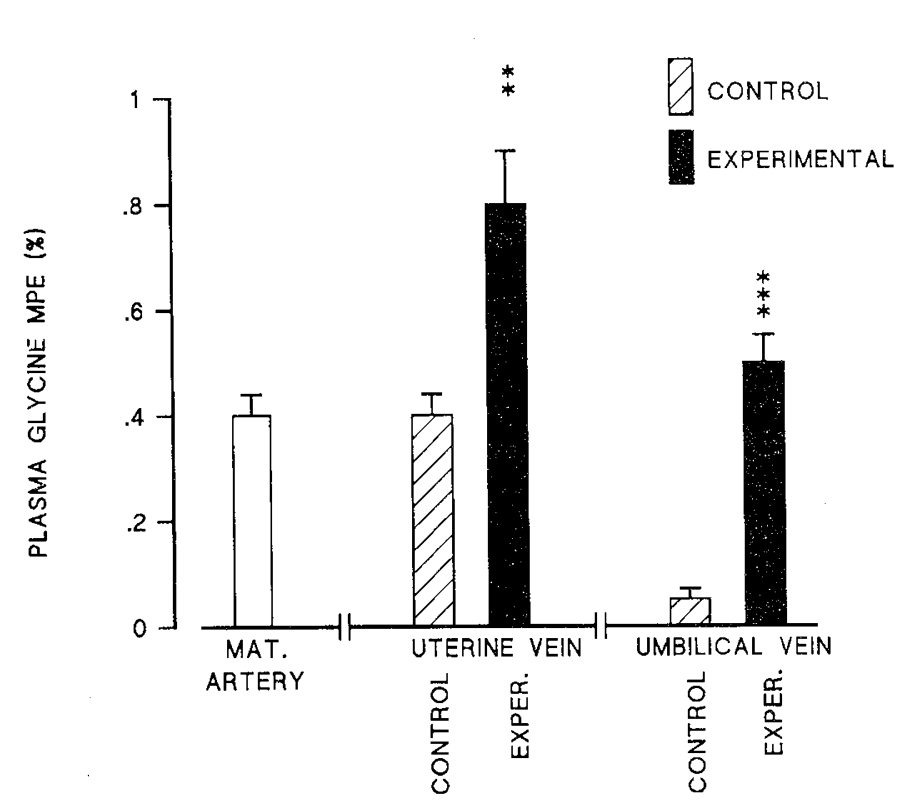

Fig. 3. Comparison of plasma glycine enrichments in systemic maternal arterial, uterine venous, and umbilical venous blood during continuous infusion of tracer serine in the maternal circulation of the experimental placenta. Experimental vs control, ${ }^{* *}, p<0.01,{ }^{* * *}, p<$ 0.001 .

$$
q_{f}=\left(r_{i}^{x}-r_{a}^{x}\right) /\left(v^{x}-a^{x}\right)
$$

For uterine blood flow to each horn:

$$
Q_{m}=\left(r_{i}^{x}-r_{a}^{x}\right) /\left(V^{x}-A^{x}\right)
$$

where $\mathrm{q}_{\mathrm{f}}$ and $\mathrm{Q}_{\mathrm{m}}$ are umbilical and uterine blood flows, respectively, to that uterine horn containing the fetus infused with substance "x."

$$
r_{i}^{x}=\text { rate of infusion of " } \mathrm{x} \text { " }
$$

$$
r_{a}^{x}=\text { rate of accumulation of } \mathrm{x} \text { in fetal body water }
$$

Oxygen uptake by the fetal circulation was calculated by application of the Fick principle. Tracer $\left[1-{ }^{13} \mathrm{C}\right]$ serine $\left(\mathrm{Ser}^{13}\right)$ and $[1-$ ${ }^{13} \mathrm{C}$ leucine $\left(\mathrm{Leu}^{13}\right)$ concentrations were calculated from the plasma serine $\left(\mathrm{Ser}^{\mathrm{C}}\right)$ and leucine $\left(\mathrm{Leu}^{\mathrm{C}}\right)$ concentrations and their plasma enrichments:

$$
\begin{aligned}
\operatorname{Ser}^{13} & =\operatorname{Ser}^{M P E} \times \operatorname{Ser}^{C} \times 0.01 \\
\operatorname{Leu}^{13} & =\operatorname{Leu}^{M P E} \times \operatorname{Leu}^{C} \times 0.01
\end{aligned}
$$

Statistics. The results are presented as mean \pm SEM. MannWhitney $t$ test was used to calculate the significance of differences in enrichments between vessels.

\section{RESULTS}

Mean \pm SEM fetal weights, blood flows, and oxygen consumption are presented in Table 1 . These data are consistent with previous studies in healthy, late-gestation fetal sheep. None 
PLASMA SERINE \& GLYCINE ENRICHMENTS

UMBILICAL CIRCULATION

UTERINE CIRCULATION
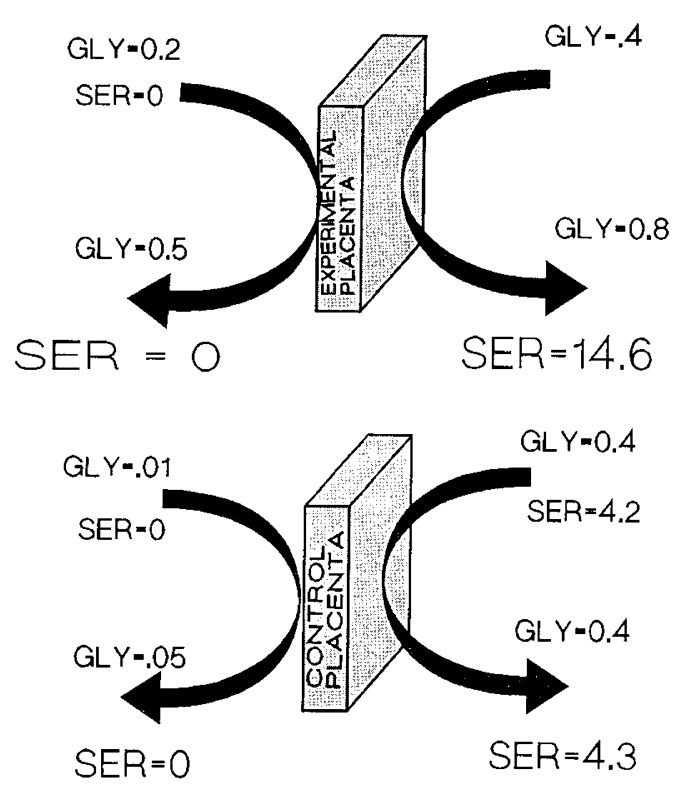

Fig. 4. Summary of plasma serine $(S E R)$ and glycine $(G L Y)$ enrichments in the four vessels supplying and draining the control and experimental placentas during exposure of the maternal surface of the experimental placenta to a high concentration of tracer serine.

of the values were significantly different for the experimental and control uterine horns.

Leucine enrichment. Leucine enrichment in the uterine vein draining the experimental horn of the uterus was $5.8 \pm 0.5 \%$ compared with $3.4 \pm 1.2 \%$ in the uterine vein draining the control horn. Leucine enrichments in the umbilical venous plasma of the experimental and control fetuses were $3.2 \pm 0.3$ and $1.7 \pm 0.4 \%$, respectively (Table 2 ). The ratios of fetal to maternal venous leucine enrichments were not significantly different between the experimental and control fetuses.

Serine and glycine enrichments. Serine and glycine data for the three animals are summarized in Table 2. Figure 2 presents the serine and glycine enrichments for a single experiment. During the ${ }^{13} \mathrm{C}$-labeled serine infusion, there was a significant ${ }^{13} \mathrm{C}$-labeling of glycine in both the maternal and fetal circulations. By contrast, there was no detectable fetal serine enrichment in either fetal circulation, despite a 280 -min labeled serine infusion that elevated the serine enrichment in the uterine vein of the experimental horn to approximately $18 \%$. Glycine enrichments in the experimental uterine and umbilical veins were significantly greater than in their corresponding control vessels $(p<0.01$ uterine and $p<0.001$ umbilical). Furthermore, in the experimental uterine horn, there were significant arteriovenous differences of glycine enrichment across both the uterine $(p<0.001)$ and umbilical $(p<0.02)$ circulations, with the venous enrichments significantly higher than the arterial. These data demonstrate that the uteroplacental tissues drained by the uterine and umbilical veins of the experimental horn were a site of glycine production from maternal plasma serine. Figure 3 summarizes the mean plasma glycine enrichments for the three sets of twins.

\section{DISCUSSION}

Recent studies of serine and glycine metabolism have demonstrated that the interconversion of these amino acids, their relative oxidation rates, and their transport into cells are quite different in different organs and tissues (1). For example, in adult kidney there is net serine production (18). Although glycine is utilized by the kidneys for serine production through the action of SHMT, it accounts for only a fraction of the total serine produced $(18,19)$. In adult liver, there is glycine utilization but no net serine production and an increase in mitochondrial glycine oxidation within minutes after ingestion of a high-protein meal (20). In contrast, studies in our laboratory have shown that the fetal liver is a site of net serine production and of glycine oxidation. Fetal hepatic glycine oxidation accounts for most of the $\mathrm{CO}_{2}$ derived from fetal oxidation of plasma glycine (4). By contrast, the bulk of fetal plasma serine oxidation occurs in extrahepatic tissues (7). Thus, despite the fact that serine and glycine are potentially interconvertible in all tissues, they are handled quite differently depending upon the organ.

The present study uses twin pregnancies to examine the question of whether, under normal physiologic conditions, maternal serine is delivered to the fetus via placental transport and whether it is utilized within the placenta for glycine production. To distinguish labeled glycine that was produced in the maternal organism from serine and then entered the fetal circulation via placental glycine transport versus labeled glycine produced in the placenta, we catheterized both fetuses in the two horns but infused $\mathrm{L}-\left[\mathrm{I}^{1{ }^{13}} \mathrm{C}\right] \mathrm{serine}$ only into the uterine artery supplying one horn. This model has a deficiency in that we cannot sample the maternal arterial blood in the perfused horn. Furthermore, labeled serine concentration in the blood perfusing the experimental placenta was probably uneven. Thus, it is not possible to calculate the uterine uptake of labeled serine from the maternal circulation. Despite this limitation, the approximate serine enrichment in the plasma supplying each uterine horn and placenta could be estimated from the enrichments in the venous drainage of each horn.

The plasma serine and glycine enrichments in the four vessels supplying and draining the two placentas are summarized in Figure 4. The serine enrichment in plasma samples from the uterine vein draining the study horn of the uterus was approximately 3.5 times the serine enrichment in the contralateral uterine horn, the latter representing dilution by the unlabeled maternal serine pool. Despite the high serine enrichment in the maternal circulation and a 280 -min infusion, there was no serine enrichment detected in either fetal circulation. In part, our inability to detect $\left[\mathrm{l}^{13} \mathrm{C}\right] \mathrm{serine}$ in the fetal circulation may be an analytic sensitivity problem, because the serine concentration is much higher in fetal than in maternal plasma (Table 2). This is not, however, adequate to account for the absence of fetal plasma serine enrichment because glycine, which has a fetal concentration only slightly less than serine, was significantly enriched in the fetal plasma of the experimental horn. It is clear, therefore, that maternal serine does not contribute significantly to the fetal plasma serine pool.

The second striking finding of the present study is that the maternal infusion of tracer serine leads to the appearance in the fetal circulation, not of the amino acid infused, but of its metabolic product, glycine. In interpreting these findings, it is important to note that the infusion of tracer serine into the fetus has demonstrated rapid bidirectional transport of serine between fetal blood and placenta, as well as placental conversion of fetal plasma serine to fetal glycine (7). Because placental glycine production from maternal serine implies that the maternal surface of the placenta is also permeable to serine, there is no obvious explanation for the fact that maternal serine molecules do not enter the fetal serine pool at an appreciable rate. It would seem that virtually every serine molecule that enters the placenta from its maternal surface is channeled into metabolic pathways (e.g., glycine formation, protein synthesis) before reaching the fetal surface and exchanging with fetal serine. We cannot exclude, however, more complex mechanisms. It is possible, for example, that the placental-fetal serine exchange and the conversion of maternal serine into fetal glycine take place in separate and specialized areas of the placenta, each endowed with different 
amino acid transport characteristics. Studies are needed to establish whether the human placenta is also a barrier to the transport of maternal serine. Serine has been shown to inhibit alanine (21) and tyrosine (22) transport in isolated microvesicles prepared from the maternal surface of the human trophoblast. It is not clear, however, whether the function of this transport system is to promote serine to glycine conversion within the placenta or to deliver serine into fetal blood at a rate that is physiologically significant. One might question why $\left[1-{ }^{13} \mathrm{C}\right]$ serine was not found in the fetal circulation, inasmuch as our laboratory has shown (4) that glycine is used for serine synthesis within the fetal liver and, in the present study, $\left[{ }^{13} \mathrm{C}\right]$ glycine appeared in the fetal circulation. However, in the previous study (4), plasma serine enrichment only reached $5 \%$ of fetal plasma glycine enrichment. At the umbilical venous plasma glycine enrichments found in the present study $(0.5 \%)$, serine enrichment would equal $0.025 \%$, which is well below the limits of detection.

The present study is the first in vivo demonstration of the placental conversion of one maternally supplied amino acid to another. Presumably, the pathway for glycine production from serine is through SHMT. A recent study has shown that ovine placental tissue has moderately high activity for the mitochondrial isoform of this enzyme, but lacks any cytosolic SHMT activity (23). An important by-product of placental glycine production via SHMT is the production of equimolar quantities of methylene tetrahydrofolate within placental mitochondria.

\section{REFERENCES}

1. Jungas RL Halperin ML, Brosnan JT 1992 Quantitative analysis of amino acid oxidation and related gluconeogenesis in humans. Physiol Rev 72:419448

2. Holzman IR, Lemons JA, Meschia G, Battaglia FC 1979 Uterine uptake of amino acids and placental glutamine-glutamate balance in the pregnant ewe. J Dev Physiol 1:137-149

3. Lemons JA, Schreiner RL 1983 Amino acid metabolism in the ovine fetus. Am J Physiol 244:E459-E466

4. Cetin I, Fennessey PV, Quick AN, Marconi AM, Meschia G, Battaglia FC, Sparks JW 1991 Glycine turnover and oxidation and hepatic serine synthesis from glycine in fetal lambs. Am J Physiol 260:E371-E378
5. Morriss FH, Adcock EW, Paxson CL, Greeley WJ 1979 Uterine uptake of amino acids throughout gestation in the unstressed ewe. Am $\mathrm{J}$ Obstet Gynecol 135:601-608

6. Lemons JA, Schreiner RL 1984 Metabolic balance of the ovine fetus during the fed and fasted states. Ann Nutr Metab 28:268-280

7. Cetin I, Fennessey PV, Sparks JW, Meschia G, Battaglia FC 1992 Fetal serine fluxes across the fetal liver, hindlimb and placenta in late gestation: the role of fetal serine in placenta glycine production. Am J Physiol 263:E786-E793

8. Moores RR, Carter BS, Meschia G, Quick AN, Delmonte S, Fennessey PV, Battaglia FC 1990 Serine metabolism in the mid-gestation fetal lamb. Clinical Res 38:171A(abstr)

9. van Veen LCP, Meschia G, Hay Jr WW, Battaglia FC 1987 Leucine disposal and oxidation rates in the fetal lamb. Metabolism 36:48-53

10. Loy GL, Quick Jr AN, Hay Jr WW, Meschia G, Battaglia FC, Fennessey PV 1990 Fetoplacental deamination and decarboxylation of leucine. Am J Physiol 259:E492-E497

11. Liechty EA, Denne SC, Lemons JA, Kien CL 1991 Effects of glucose infusion on leucine transamination and oxidation in the ovine fetus. Pediatr Res 30;423-429

12. Wilkening RB, Anderson S, Martensson L, Meschia G 1982 Placental transfer as a function of uterine blood flow. Am J Physiol 242:H429-H436

13. Bonds DR, Anderson S, Meschia G 1980 Transplacental diffusion of ethanol under stready-state conditions. J Dev Physiol 2:409-416

14. van Veen LCP, Hay Jr WW, Battaglia FC, Meschia G 1984 Fetal $\mathrm{CO}_{2}$ kinetics. J Dev Physiol 6:359-365

15. Wilkening RB, Anderson S, Martensson L, Meschia G 1982 Placental transfer as a function of uterine blood flow. Am J Physiol 242:H429-H436

16. Marconi AM, Battaglia FC, Meschia G, Sparks JW 1989 A comparison of amino acid arteriovenous differences across the liver and placenta of the fetal lamb. Am J Physiol 257:E909-E915

17. Meschia G, Cotter JR, Makowski EL, Barron DH 1966 Simultaneous measurement of uterine and umbilical blood flows and oxygen uptakes. Q J Exp Physiol 52:1-18

18. Lowry M, Hall DE, Hail MS, Brosnan JT 1987 Renal metabolism of amino acids in vivo: studies on serine and glycine fluxes. Am J Physiol 252:F304F309

19. Lowry M, Hall DE, Brosnan JT 1986 Serine synthesis in rat kidney: studies with perfused kidney and cortical tubules. Am J Physiol 250:F649-F658

20. Ewart HS, Jois M, Brosnan JT 1992 Rapid stimulation of the hepatic glycinecleavage system in rats fed on a single high-protein meal. Biochem J 283:441447

21. Hoeltzli SD, Smith CH 1989 Alanine transport systems in isolated basal plasma membrane of human placenta. Am J Physiol 256:C630-C637

22. Kudo Y, Body CAR 1990 Human placental l-tyrosine transport: a comparison of brush-border and basal membrane vesicles. J Physiol 426:381-395

23. Moores RR, Frerman FE, Watmough NJ, Battaglia FC 1990 Enzymatic basis of placental and fetal hepatic serine/glycine fluxes: I. Serine hydroxymethyltransferase. Pediatr Res 27:48A 